\title{
New horizons in hydrogen bonded clusters in solution*
}

\author{
Toshio Yamaguchi \\ Department of Chemistry, Faculty of Science, Fukuoka University, Fukuoka 814-0180, \\ Japan
}

\begin{abstract}
Breakthroughs in X-ray and neutron diffraction of liquids and solutions by the advent of intense synchrotron radiation, pulsed neutron sources, and an area detector of imaging plate over the last two decades have enabled us to manifest the structures of hydrogen-bonded clusters in solution on the molecular level over a wide range of temperature and pressure covering the supercritical state. Furthermore, computer simulation techniques with empirical potentials based on experiments have revealed the orientational correlation and threedimensional pictures of hydrogen bonded clusters in solution. The microscopic structures manifested by using these techniques have been described as examples of methanol and ethanol-water binary solutions from ambient to the supercritical state. The roles of hydrogenbonded clusters have been demonstrated for micelle formation of non ionic surfactant MEGA8 and alcohol-induced $\alpha$-helix formation of chymotrypsin inhibitor 2 in aqueous mixtures of ethanol.
\end{abstract}

\section{INTRODUCTION}

Hydrogen bonded clusters, such as water clusters, have been implicated in various problems in many fields, including the formation of acid rain and the nucleation of water droplets in environmental science, conservation of living cells in cryobiology, destruction of chlorinated organic wastes in supercritical water technology as well as aqueous solvation, cooperativity in hydrogen bonding, etc. in physics and chemistry [1,2]. A surge of progress in X-ray and neutron diffraction experiments and computer simulations has facilitated new, highly detailed studies of hydrogen bonded clusters in condensed phases under various conditions. In this article, we describe some of these developments and the structural insight that has been gained from them.

\section{BREAKTHROUGHS IN DIFFRACTION METHODS}

\section{$X$-ray and neutron diffraction}

A diffraction method using X-rays and neutrons has been successfully used to determine the microscopic structures of hydrogen bonded liquids and solutions, such as water [3] and aqueous solutions [4,5], since it provides us direct structure information (interatomic distance and coordination number) at a molecular level in terms of radial distribution functions. Concerning liquid X-ray diffraction, however, a conventional $\theta-\theta$ type of X-ray diffraction combined with a sealed X-ray tube and a scintillation counter usually needs long measuring time $(150 \sim 300 \mathrm{~h}$ per sample) to obtain X-ray scattering intensities of high quality; thus, it was difficult or sometimes impossible to perform X-ray diffraction measurements of liquids and solutions under extreme conditions, such as high-temperatures and high-pressures. In the last decade, intense X-rays $\left(10^{2} \sim 10^{3}\right.$ times for bending magnet beams and $10^{4} \sim 10^{5}$ times for undulator beams to those of laboratory $\mathrm{X}$-rays) became available in synchrotron radiation facilities world-wide, and

\footnotetext{
* Plenary lecture presented at the 26th International Conference on Solution Chemistry, Fukuoka, Japan, 26-31 July 1999, pp. 1691-1764.
} 
at the same time a novel two-dimensional X-ray detector of photostimulable phosphor film, so-called imaging plate (IP), of wide dynamic range $\left(1: 10^{5}\right)$ was developed [6]. These two developments in X-ray diffraction have drastically shortened the measurement time to several minutes per liquid sample $(\sim 1 \mathrm{~h}$ when a rotatory X-ray generator in a laboratory scale is used) and thus enabled us to measure very reactive hydrogen bonded liquids and solutions, such as supercritical water [7]. At the moment the maximum temperature and pressure measured for hydrogen bonded liquid systems are $\sim 773 \mathrm{~K}$ and $\sim 700 \mathrm{MPa}$, respectively.

Another advent in the diffraction methods is neutron diffraction with $\mathrm{H} / \mathrm{D}$ isotopic substitution [4], which enables us to extract the partial structure factors related to hydrogen atom of hydrogen bonded clusters in solution. This technique utilises different scattering lengths of chemically identical isotopes $\left(-3.4 \mathrm{fm}\right.$ for $\mathrm{H}$ and $+6.3 \mathrm{fm}$ for $\mathrm{D}$ ), e.g. when three neutron diffraction experiments for $\mathrm{D}_{2} \mathrm{O}, \mathrm{H}_{2} \mathrm{O}$, and $\mathrm{D}_{2} \mathrm{O} / \mathrm{H}_{2} \mathrm{O}$ mixture are performed, the partial structure factors $\mathrm{HH}, \mathrm{OH}$ and $\mathrm{OO}$ are obtained [8]. It should be borne in mind that intense pulsed neutron sources, such as ISIS in UK, are essential to extract reliable partial structure factors routinely for complicated hydrogen bonded systems.

\section{Empirical potential structure refinement (EPSR)}

Since structure information on hydrogen bonded clusters in solution obtained from X-ray and neutron diffraction methods is one-dimensional site-site distribution functions of the system, it is often difficult to construct a three-dimensional picture of clusters and orientational correlation between molecules in the clusters. Since 1970s computer experiments, such as molecular dynamics and Monte Carlo simulations, have made a great contribution to understand three-dimensional structure and dynamics of hydrogen bonded clusters in solution [9]; however, the outcomes from the computer simulations depend fully on the pair-potentials to describe the intermolecular interactions in the system, and thus should be compared with experiments. In 1988 McGreevy developed a reverse Monte Carlo simulation method [10], which pursues three-dimensional distributions of molecules consistent with the diffraction data supplied. In 1994, Soper extended a spherical harmonic expansion [11], which was originally developed by Gray \& Gubbins [12] in 1986, to complex hydrogen bonded systems on the basis of neutron first-order difference data to extract the orientational correlation function of molecular distributions. In 1996 Soper invented an empirical potential structure refinement (EPSR) method [13], which employs a pseudo potential perturbed from a reference potential on the basis of experiment in Monte Carlo simulation to obtain consistency between the simulation and experiment.

The EPSR method can be outlined below. First, a computer simulation of the system is performed with an assumed set of site-site interaction potentials, $U_{\mathrm{ij}}{ }^{\circ}(r)$ as a function of the separation $r$ of pairs of atoms 'i' and ' $\mathrm{j}$ ', consisting of Lennard-Jones plus Coulomb potential, an internal harmonic potential on molecular geometries, and repulsive-core potential. When equilibrium is established in this initial simulation, a potential of mean force is calculated from the simulation data as $y_{\mathrm{ij}}(r)=-k T \ln \left(g_{\mathrm{ij}}(r)\right)$. A similar potential of mean force is also derived from experimental diffraction data as $y_{\mathrm{ij}}{ }^{\mathrm{E}}(r)=-k T$ $\ln \left(g_{\mathrm{ij}}^{\mathrm{E}}(r)\right)$. Then, a new interaction potential, $U_{\mathrm{ij}}^{\mathrm{N}}(r)$, is calculated as a perturbation to the initial potential; namely $U_{\mathrm{ij}}{ }^{\mathrm{N}}(r)=U_{\mathrm{ij}}{ }^{\circ}(r)+\left(y_{\mathrm{ij}} \mathrm{E}^{\mathrm{E}}(r)-y_{\mathrm{ij}}(r)\right)=U_{\mathrm{ij}}{ }^{\circ}(r)+k T\left[\ln \left\{g_{\mathrm{ij}}(r) / g_{\mathrm{ij}}{ }^{\mathrm{E}}(r)\right\}\right]$. This new set of potentials is used in the simulation, and the run continues until equilibrium is reached again. Individual site-site distribution functions are not generally obtained from X-ray and neutron diffraction, thus, the corresponding empirical potential will be a composite potential as a sum of several site-site terms. Furthermore, the experimental site-site distribution functions should be as smooth as possible to exclude experimental uncertainties in the modified potentials.

\section{RESULTS AND DISCUSSION}

\section{Cluster formation in supercritical methanol}

Hydrogen-bonded liquids such as water and methanol in the supercritical state have unique properties not available under ambient conditions and have recently attracted much attention not only in fundamental physics and chemistry but also in supercritical fluid technology as a novel reaction medium in decomposition of hazardous organic wastes like dioxine, polychlorobiphenyl (PCB), etc., and in recovery of useful chemicals from industrial plastic wastes like polyethyleneterephthalate (PET) [14]. These 
characteristic properties of supercritical hydrogen-bonded fluids and chemical reactions taking place in them originate from the microscopic structure related to hydrogen bonding, cluster formation, ion solvation, and ion association.

In order to clarify the structural details of hydrogen bonded clusters in supercritical methanol $\left(T_{\mathrm{c}}=513 \mathrm{~K}, P_{\mathrm{c}}=8.1 \mathrm{MPa}, r_{\mathrm{c}}=0.272 \mathrm{~g} / \mathrm{cm}^{3}\right)$, neutron diffraction experiments on supercritical methanol with $\mathrm{H} / \mathrm{D}$ isotope substitution on the hydroxyl hydrogens have been performed to derive the partial structure factors and the partial radial distribution functions, $\mathrm{XX}, \mathrm{XH}$ and $\mathrm{HH}$, where $\mathrm{X}$ represents a weighted sum of correlations from carbon $(\mathrm{C})$, oxygen $(\mathrm{O})$, and methyl hydrogen $(\mathrm{M})$ atoms on a methanol molecule [15]. The hydroxyl-hydrogen radial distribution functions for liquid methanol as a function of temperature (Fig. 1), show that as the temperature increases, the first peak become better defined and separates itself from the structure at larger distances. It has been found from analysis of the first peak that in supercritical methanol of moderate density $\left(526 \mathrm{~K}, 0.7 \mathrm{~g} / \mathrm{cm}^{3}\right)$ clusters with $3 \sim 5$ methanol molecules are formed, in contrast with chain-like hydrogen bonded clusters at low and ambient temperatures [16].

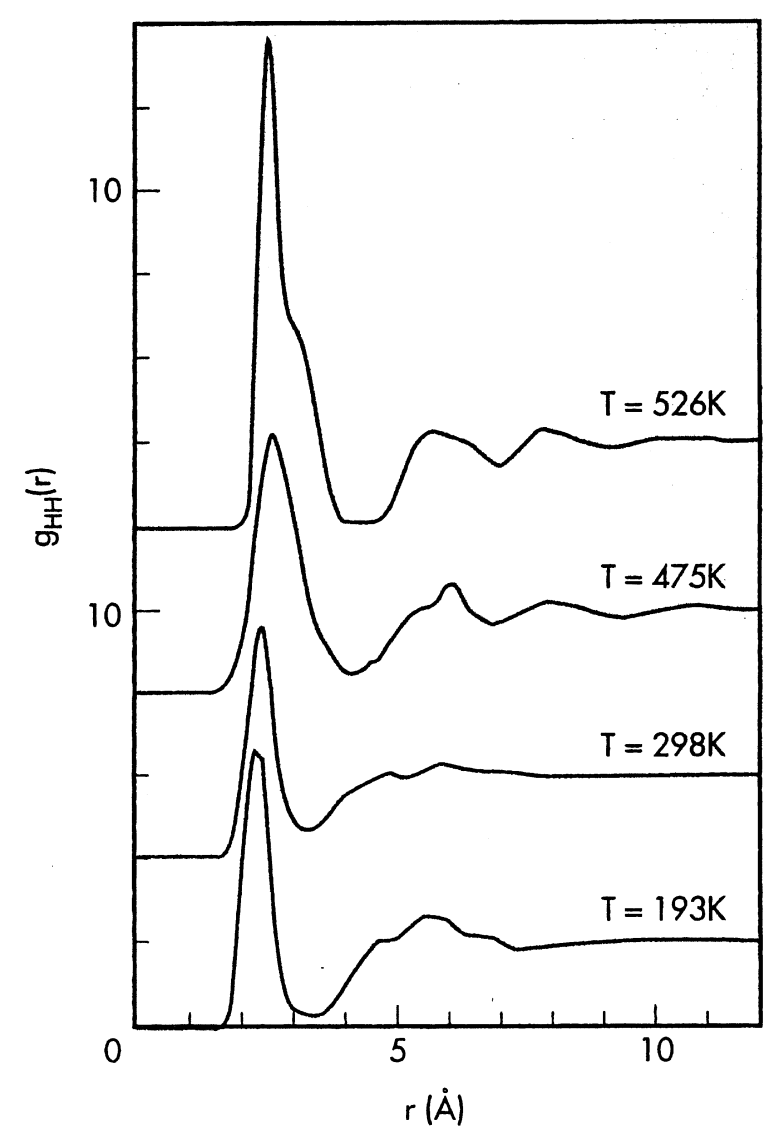

Fig. 1 The hydroxyl hydrogen radial distribution functions for methanol as a function of temperature.

The neutron partial structure factors were then used in an Empirical Potential Structure Refinement (EPSR) computer simulation. Model distributions of molecules consistent with these data were used to estimate the individual site-site radial distribution functions, the coefficients of the spherical harmonic expansion of the orientational pair correlation function, the details of hydrogen bonding, and threedimensional structure of clusters formed in supercritical methanol. At the supercritical state of the moderate density, the hydrogen bonds remain with the average number of hydrogen bonds of $1.6 \pm 0.1$ per molecule and the average chain-length of $3.1 \pm 0.4$ molecules with a part of chains up to 12 , which are less than $1.77 \pm 0.07$ and $5.5 \pm 1.0$, respectively, found under ambient conditions. In the low-density supercritical methanol $\left(526 \mathrm{~K}, 0.45 \mathrm{~g} / \mathrm{cm}^{3}\right)$, the large clusters are broken to generate monomers or small 
oligomers; the average number of hydrogen bonds per molecule decreases to $1.0 \pm 0.1$, and the chainlength is $1.8 \pm 0.2$ with a maximum length up to 7 .

The orientational pair correlation functions (OPCFs), $g\left(r, \theta_{\mathrm{L}}, \phi_{\mathrm{L}}, \phi_{\mathrm{M}}, \theta_{\mathrm{M}}, \chi_{\mathrm{M}}\right)$, in which the coordinates of molecular orientation is defined as in Fig. 2, were obtained from the coefficients of the spherical harmonic expansion. Figure 3 shows the torsional angle distribution of the neighbouring molecules as a function of $\chi_{\mathrm{M}}$ at the orientation $\theta_{\mathrm{L}}=74^{\circ}, \phi_{\mathrm{L}}=0^{\circ}, \phi_{\mathrm{M}}=0^{\circ}$, and $\theta_{\mathrm{M}}=72^{\circ}$. If the molecules formed a linear chain, these torsional angle would be peaked at $180^{\circ}$, whereas a peak at $0^{\circ}$ or $360^{\circ}$ would mean a ring structure. In the low-temperature methanol, there is little structure in this distribution, indicating a wide range of torsional angles, so that disordered or winding chains of methanol molecules form in the liquid. In the low-density supercritical methanol, however, the distribution peaks at $0^{\circ}$ or $360^{\circ}$, indicating that the chains are more likely to curl back on themselves to form small ring-like structure. It is anticipated that monomers and/or small oligomers play an important role in chemical reactions in supercritical methanol.

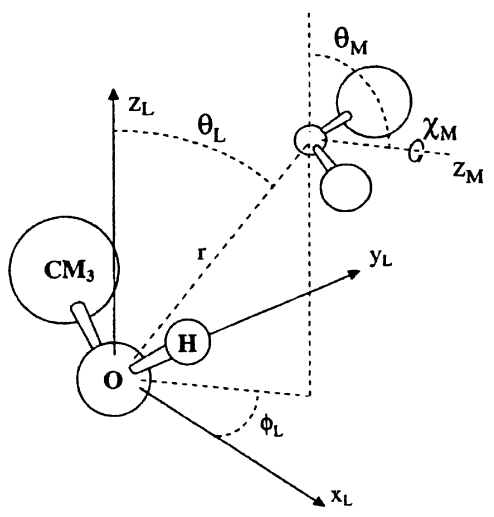

Fig. 2 Coordinate system for describing the orientational correlations of one methanol molecule about another. The $\mathrm{COH}$ plane coincides with the $x z$ plane, and $z$ axis pointing in the direction of the methanol dipole moment vector.

\section{Clusters in organic solvent-water mixtures}

Binary solutions of water and organic solvents are used in various chemical reactions, solvent extraction, high-performance liquid chromatography, a recent supercritical water technology, etc., since the solution properties can be varied by changing solvent composition. Among a large number of thermodynamic and physicochemical data of aqueous solvent mixtures so far compiled, there often appear anomalies at characteristic solvent compositions. Although various spectroscopic investigations have been carried out on aqueous solvent mixtures, few reports have clarified the anomalies and related cluster structures of aqueous binary solvents at the molecular level. We have employed both rapid X-ray diffraction method with an imaging plate detector and mass spectrometry to measure several aqueous organic mixtures, such as ethanol-water [17,18], acetonitrile-water [19], and 1,4-dioxane-water [20], and revealed how the structures of solvent clusters change with solvent compositions in connection with the anomalies.

Figure 4 shows mass spectra of the clusters, represented as $\mathrm{E}_{m} \mathrm{~W}_{n}$ (E: ethanol, W: water), isolated from ethanol-water binary solutions with the ethanol mole fraction $x_{\mathrm{E}}=0.8,0.5$, and 0.2 . In the mass region lower than 360, the main signals are pure ethanol clusters, $\mathrm{E}_{m}(m<8)$, while for $m \geq 8$, the intensities of the pure ethanol clusters become weaker relative to those of the hydrate species of $\mathrm{E}_{m} \mathrm{~W}_{n}$. It is interesting to note that the hydration number $(n)$ of the strongest signal in the hydration sequence of $\mathrm{E}_{11} \mathrm{~W}_{n}$ shifted from 2 at $x_{\mathrm{E}}=0.8$ to 3 at $x_{\mathrm{E}}=0.5-0.2$ even though the water-to-ethanol ratio at $x_{\mathrm{E}}=0.2$ is 16 times larger than that at $x_{\mathrm{E}}=0.8$. Figure 5 shows the average water numbers $\left(N^{\mathrm{a}}\right)$ as a function of $x_{\mathrm{E}}$ for ethanol $m$-mer hydrates with $m=6-14$. The $N^{\mathrm{a}}$ value in the clusters linearly decreases with increasing $x_{\mathrm{E}}$ from 0.2 to 0.9 , while at $x_{\mathrm{E}}<0.2$ the average water numbers change drastically. Thus, the solvent clusters are likely to change at $x_{\mathrm{E}}=0.2$. 


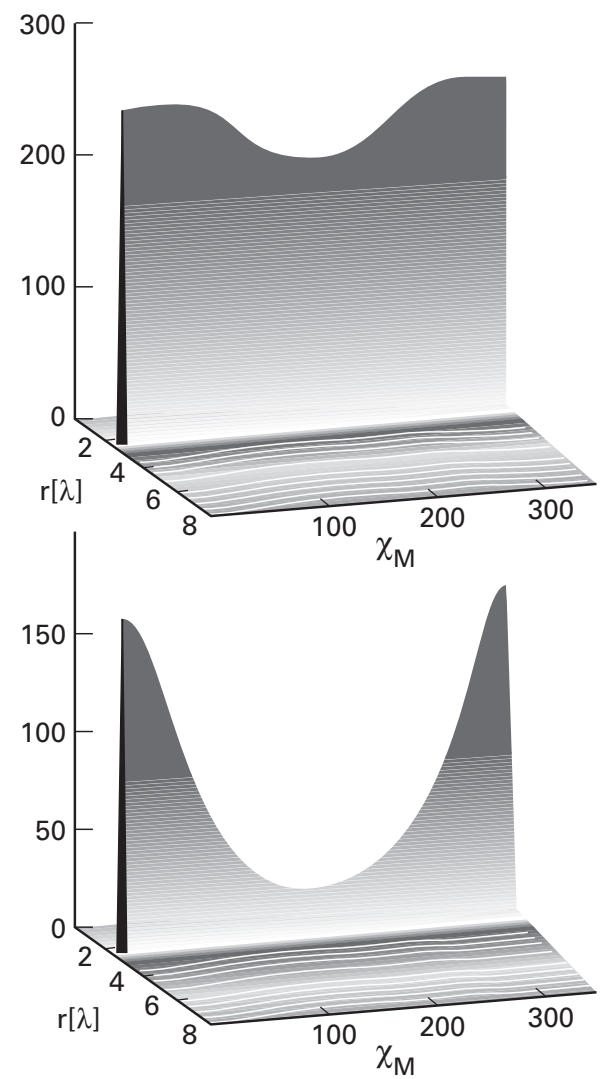

Fig. 3 The OPCFs show distribution of torsional angles $\chi_{M}$ for a neighbouring pair of methanol molecules. Top: $193 \mathrm{~K}$, bottom: $526 \mathrm{~K}$.

$\mathrm{X}$-ray radial distribution functions (RDFs) of ethanol-water mixtures have revealed the details of structure change in clusters as a function of $x_{\mathrm{E}}$ (see Fig. 6). In all of the RDFs, the intramolecular $\mathrm{C}-\mathrm{O}$ and $\mathrm{C}-\mathrm{C}$ bonds and $\mathrm{C}\left(\mathrm{CH}_{3}\right)-\mathrm{O}$ interactions in ethanol appear at 1.5 and $2.5 \AA$, respectively. The features of RDFs beyond $2.5 \AA$ correspond to intermolecular structure of clusters; the $2.8 \AA$ peak characterises the $\mathrm{O}-\mathrm{O}$ hydrogen bonds between water-water, water-ethanol, and ethanol-ethanol. From geometrical consideration, the $3.8 \AA$ peak in pure ethanol were assigned to $\mathrm{O}-\mathrm{C}$ distance of the hydrogen bonded ethanol molecules, and the $4.6 \AA$ peak to the methylene-methylene and methyl-methyl distances. In pure water, the tetrahedral-like structure is characterised by three prominent peaks at $2.8,4.5$, and $6.8 \AA$ due to the first-, second-, and third-nearest neighbour water-water interactions. It is clearly seen that the clusters change with increasing ethanol fraction; typically the water structure is drastically broken down up to $x_{\mathrm{E}}=\sim 0.2$ as evidenced by a sharp decrease of the $2.8 \AA$ peak from $x_{\mathrm{E}}=0.0-0.2$, but at $x_{\mathrm{E}}>0.2$ the cluster gradually change with increasing $x_{\mathrm{E}}$. From the mass spectra and $\mathrm{X}$-ray data the most likely cluster models for ethanol-water mixtures are proposed in Fig. 7; at $x_{\mathrm{E}}<0.2$, the ethyl groups form a stacked hydrophobic core, which is coupled with an interfacial water layer of linear hydrogen bonding (LHB). The outer bulk water area consists of non linear hydrogen bonds (NLHB) and LHB. At $0.2 \leq x_{\mathrm{E}} \leq 0.4$, water molecules serve as stabilisers for ethanol polymer clusters.

\section{Micelle formation in ethanol-water solvents}

Surfactants have amphiphile substances with both hydrophilic and hydrophobic groups and form micelles above the critical micelle concentration (CMC). The structure and properties of micelles of ionic and nonionic surfactants have been investigated in aqueous media by various methods; however, very few studies have been performed in aqueous mixtures of organic solvents since micelles are decomposed by addition of organic solvents, such as alcohols. Surfactants can be regarded as a simple model of complex biomembranes with amphiphile properties. 


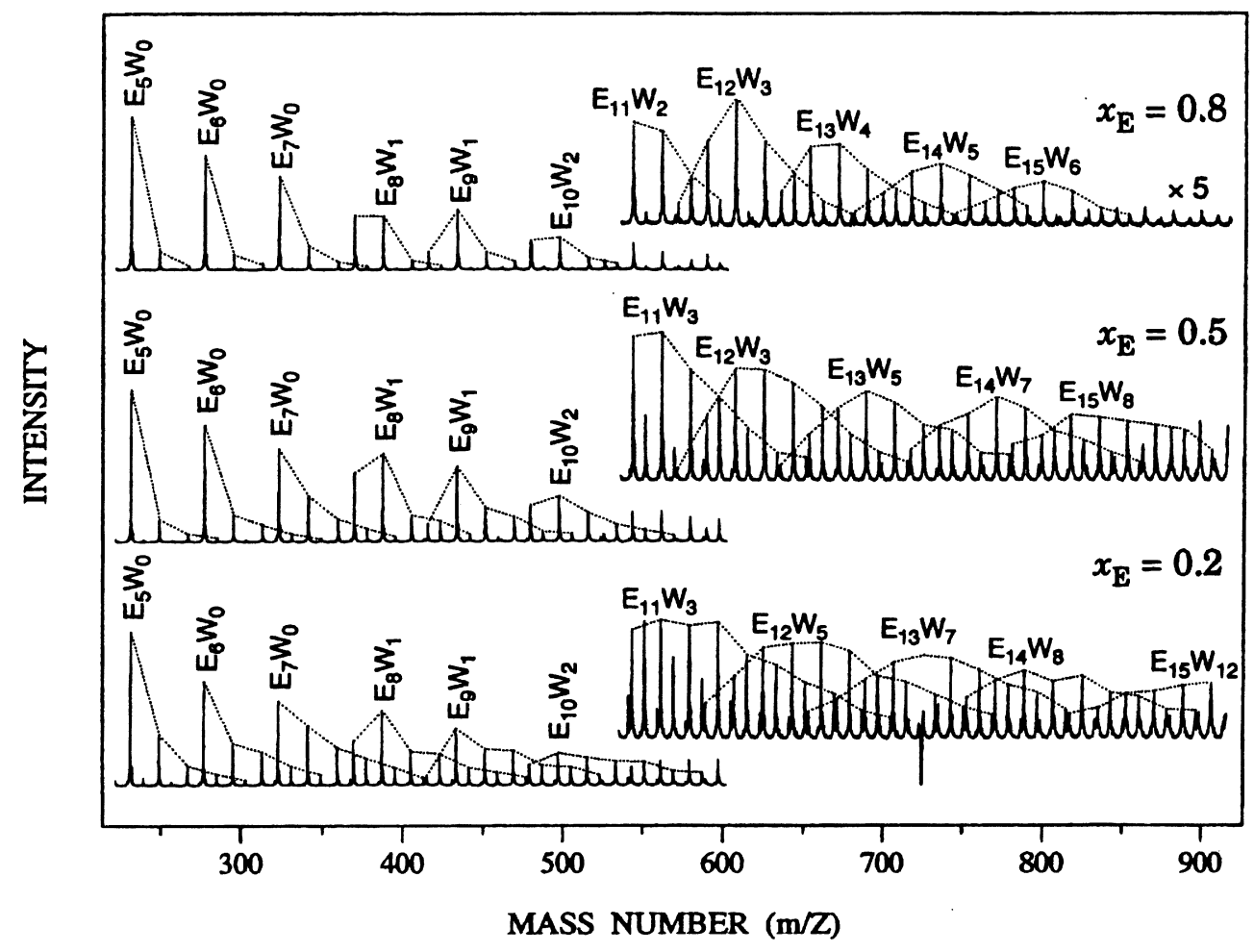

Fig. 4 Mass spectra of the clusters isolated from ethanol-water binary solutions with $x_{\mathrm{E}}=0.8,0.5$, and 0.2.

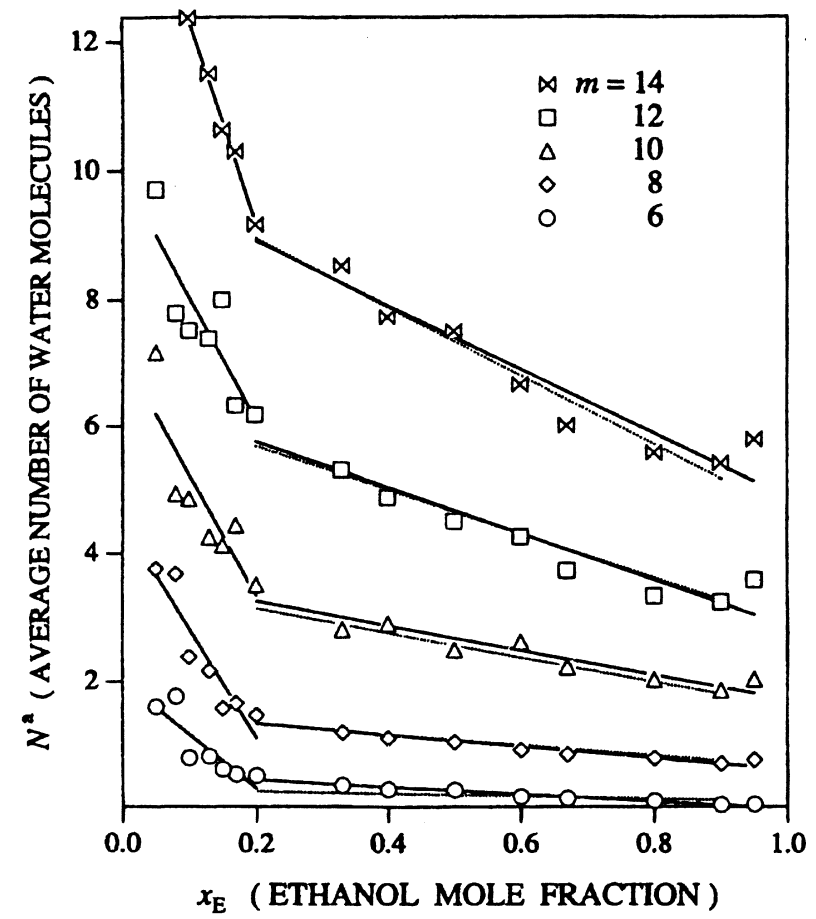

Fig. 5 Plots of average water numbers $\left(N^{\mathrm{a}}\right)$ as a function of $\mathrm{xE}$ for ethanol $m$-mer hydrates. The dotted lines are obtained by $N^{\mathrm{a}} \approx\{(m / 4.2)-1]^{2}\left(x_{\mathrm{w}}+0.85\right)$. 


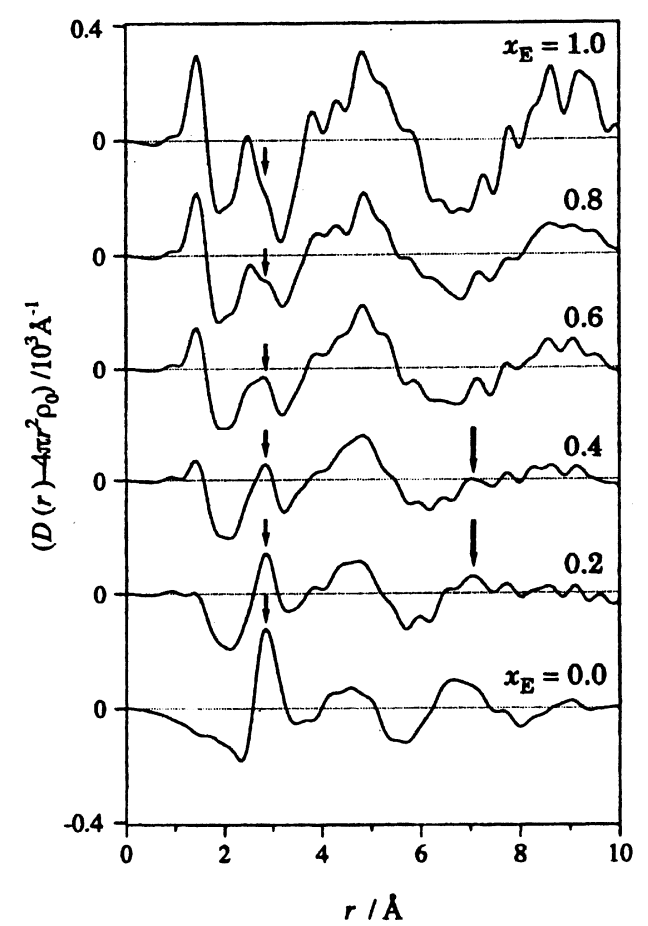

Fig. $6 \mathrm{X}$-ray radial distribution functions for ethanol-water binary solutions as a function of $x_{\mathrm{E}}$.
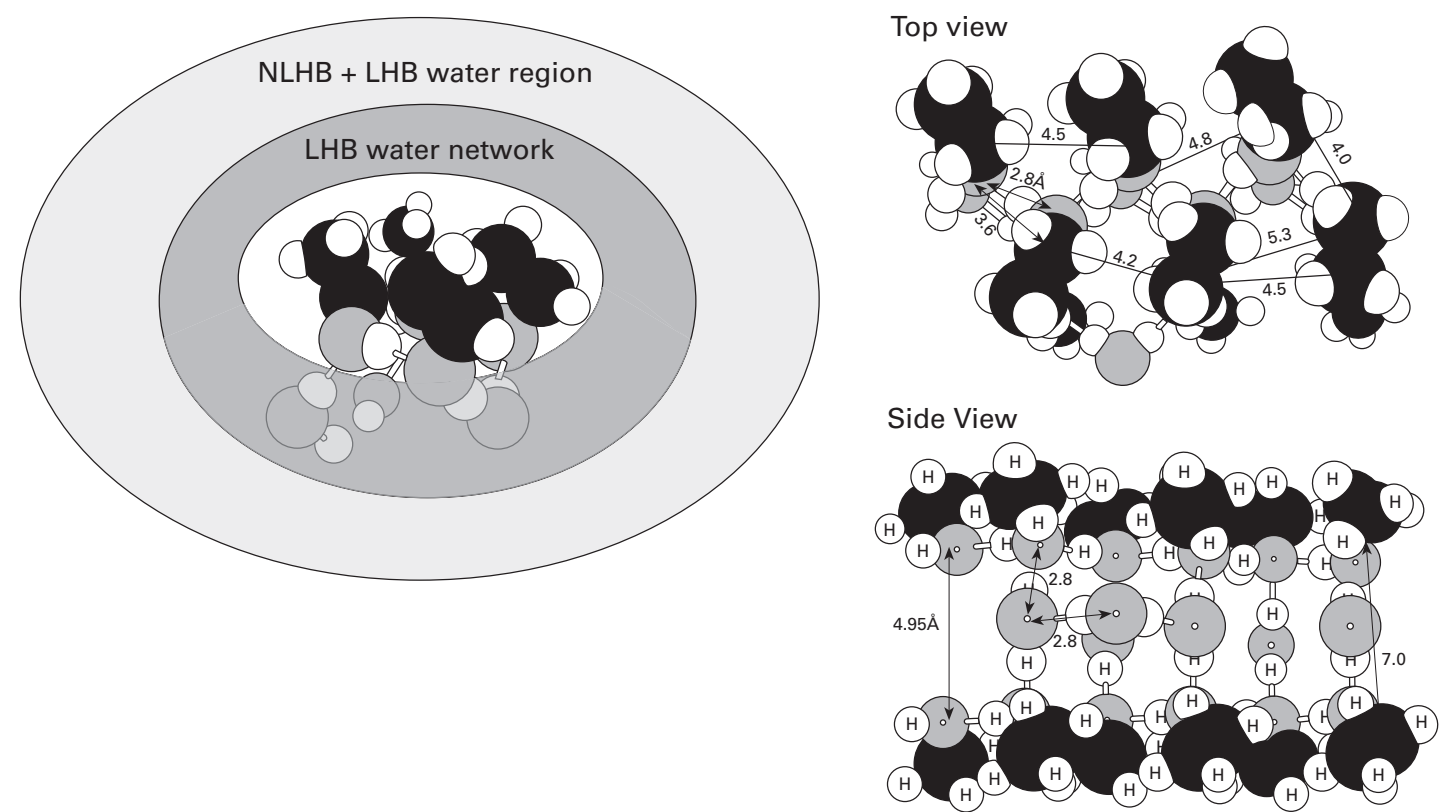

Fig. 7 Models of clusters formed in ethanol-water constructed based on the mass spectra and X-ray data in the regions $0<\mathrm{x}_{\mathrm{E}}<0.2$ (left) and $0.2 \leq \mathrm{x}_{\mathrm{E}} \leq 0.4$ (right).

$N$-octanoyl- $N$-methylglucamide, abbreviated as MEGA- 8 , is a nonionic surfactant of five hydroxyl groups, and thus its CMC is as high as $0.065 \mathrm{~mol} / \mathrm{kg}$, comparable with those for ionic surfactants. We have investigated micelle formation of MEGA-8 in water [21] and in ethanol-water mixtures [22] by dynamic light scattering, small-angle X-ray and neutron scattering, and molecular dynamics computer simulation. Figure 8 shows the micelle size of MEGA-8 $(d)$, which is twice the hydrodynamic radius obtained from dynamic light scattering, as a function of ethanol concentration, $x_{\mathrm{a}}$, in aqueous mixtures of ethanol. As is 
seen in Fig. 8, the micelle size increases with increasing $x_{\mathrm{a}}$ and becomes largest at $x_{\mathrm{a}}=\sim 0.1$ and decreases drastically from $x_{\mathrm{a}}=\sim 0.1$ to $\sim 0.2$, and at $x_{\mathrm{a}}>\sim 0.2$ the micelles are gradually broken down. Thus the micelle formation is affected significantly by addition of ethanol and the breaking-down of the micelle occurs at $x_{\mathrm{a}}=0.1 \sim 0.2$, where the solvent structure changes from predominant water clusters to ethanol ones.

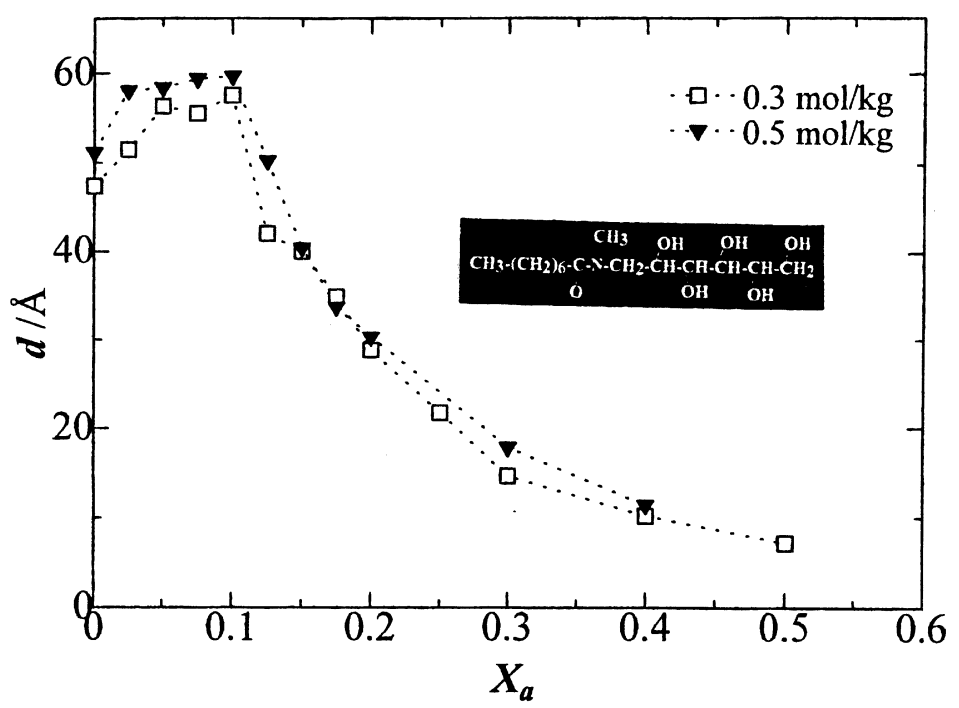

Fig. 8 The hydrodynamic diameter of MEGA-8 micelles in ethanol-water mixtures as a function of ethanol mole fraction $x_{\mathrm{a}}$.

This mechanism of micelle deformation induced by addition of alcohol might be answered from a detailed internal structure of MEGA-8 micelle in water derived from a molecular dynamic (MD) simulation. The MD simulation was performed by a Nose-Andersen $N P T$ method at $25^{\circ} \mathrm{C}$ and $1 \mathrm{~atm}$ on a unit cell containing 49 MEGA-8 molecules and 5400 water molecules. The intermolecular bonded and non bonded potentials for MEGA-8 used were AMBER and OPLS, respectively. The water potential was TIP3P. Figure 9 shows how the hydrophobic and hydrophilic groups of MEGA-8 and water molecules distribute inside the micelle. Interestingly, the ends of hydrophobic groups are widely distributed from the micelle centre to $15 \AA$. Water molecules penetrate into almost half of the micelle radius, near the $\mathrm{C}=\mathrm{O}$ group. In the case of ethanol-water mixtures, amphiphile ethanol molecules might enter the micelle and perturb a subtle hydrophobic and hydrophilic balance, leading to breaking down of the micelles. An MD simulation is being carried out on MEGA-8 in ethanol-water mixtures, from which a more definite answer will be obtained.

\section{Alcohol-induced peptide folding in ethanol-water mixtures}

Although peptides are well known to be induced to form $\alpha$-helices in water by addition of alcohols, the details of its mechanism remains unknown. Recent investigations on several peptides in aqueous mixtures of alcohols, such as 2,2,2-trifluoroethanol (TFE) and 1,1,1,3,3,3-hexafuluoro-2-propanol (HFIP), have shown that helix induction takes place only in a specific alcohol concentration region [23].

Chymotrypsin inhibitor 2 (CI2) is one of proteins investigated in detail. The structure of CI2 has been determined in solution by NMR [24] and in crystals [25] and consists of a four-stranded mixed parallel and anti-parallel $\beta$-sheet packed against an $\alpha$-helix to form the hydrophobic core (see Fig. 10). We have investigated alcohol-induced $\alpha$-helix formation in ethanol-water mixtures [26]. CI2 was synthesised with Milligen 9050 by the solid phase method using fluorenylmethoxycarbonyl (Fmoc) chemistry. The crude CI2 was purified by preparative $\mathrm{C} 18$ reverse phase HPLC, and the purity of the peptide was confirmed to be $>95 \%$ by analytical C18 reverse phase column chromatography (COSMOSIL 5C-18AR). The peptide identity was confirmed by FAB mass spectrometry. CD spectra were taken over the wavelength from 190 to $260 \mathrm{~nm}$ on an JASCO-J600 spectropolarimeter. 


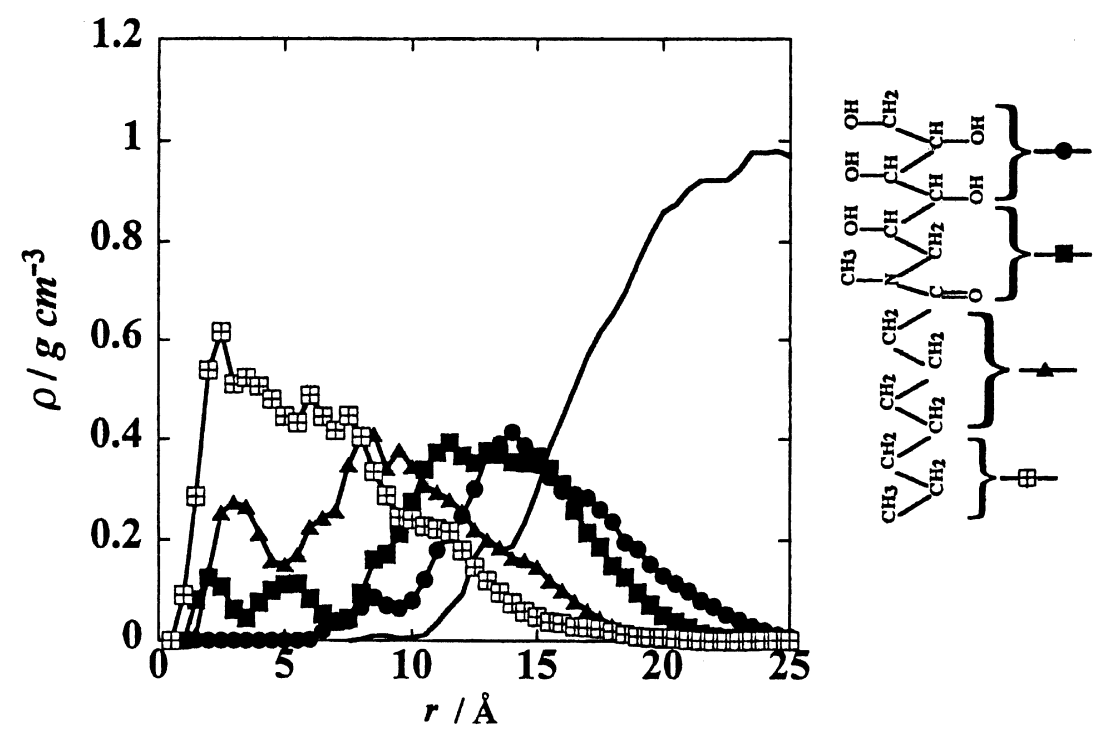

Fig. 9 MEGA-8 and water densities as a function of distance from the centre of the micelle.

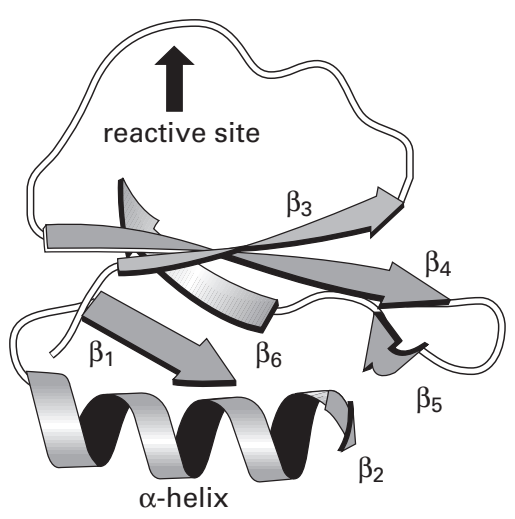

Fig. 10 Secondary structure of CI2

Figure 11 shows the CD spectra of $25 \mu \mathrm{M}$ CI2 in ethanol-water mixtures as a function of ethanol composition. In water, CI 2 contains both $\alpha$-helix and $\beta$-sheets as well as random structures. When the ethanol composition is increased, the CD spectra show typical minima at 208 and $222 \mathrm{~nm}$, characteristic for the $\alpha$-helix. The secondary structure components $(\alpha$-helix, anti-parallel and parallel $\beta$-sheet, turn structure, and random structure) were calculated by a self-consistent method applied to the CD spectra and are shown as a function of ethanol concentration in Fig. 12. With increasing ethanol content up to $\sim 0.2$, the fraction of $\alpha$-helix gradually increases, whereas that of the anti-parallel $\beta$-sheet gradually decreases; however, at $x_{\mathrm{E}}>\sim 0.2$ such increasing and decreasing tendencies of both structure become much stronger, resulting in an inflection point at $x_{\mathrm{E}}=\sim 0.2$. This solvent composition corresponds to where the solvent clusters change from tetrahedral-like water to methanol polymers. Molecular dynamic simulation and small-angle X-ray and neutron scattering measurements are in progress to look further insight into the folding mechanism from the anti-parallel $\beta$-sheet to the $\alpha$-helix from a standpoint of solvent clusters.

\section{CONCLUSIONS AND FUTURE PROSPECTS}

$\mathrm{X}$-ray diffraction and neutron diffraction with isotopic substitution have been developed in deepening our understanding of the microscopic structures of hydrogen bonded clusters in aqueous mixtures under various conditions and environments. Comparative and/or combined experiments and computer simulations have provided a detailed three dimensional picture of complex liquids. 


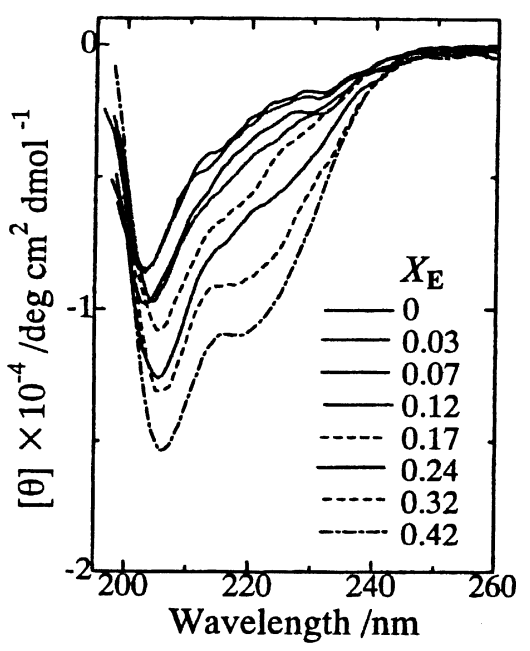

Fig. 11 CD spectra of CI2 in ethanol-water binary solutions as a function of $x_{\mathrm{E}}$. The concentration of CI2 is $25 \mu \mathrm{M}$.

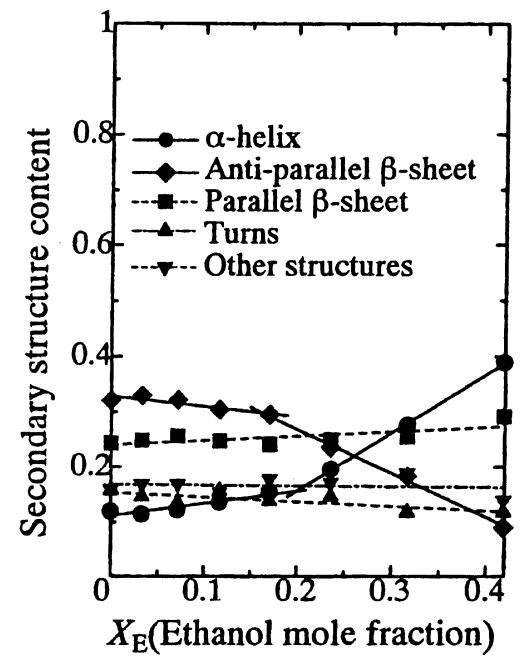

Fig. 12 Fractions of secondary structure of CI2 in ethanol-water binary solutions obtained from the selfconsistent method.

The third generation synchrotron radiation and pulsed neutron sources dedicated and under construction or planning will surely be a useful tool in the 21 st century to open new horizons of (i) hydrogen bonded clusters in a real-time scale or in nonequilibrium state, (ii) excited states of clusters when combined with laser spectroscopy, (iii) reaction intermediates to reveal reaction mechanisms, and (iv) dilute systems often encountered in biological systems.

\section{ACKNOWLEDGEMENTS}

The work presented in this paper represents a combined effort of colleagues at Fukuoka University in collaboration with the National Laboratory for High Energy Physics (KEK, Japan), Rutherford Appleton Laboratory (RAL, UK), Institute of Molecular Science (IMS, Japan), Saga University, and Tokyo Institute of Technology (T.I.Tech, Japan). I thank Dr C. J. Benmore and Prof. A. K. Soper (RAL) for neutron diffraction measurements and EPSR simulations, Prof. T. Takamuku (Saga University of) and Prof. N. Nishi (IMS) for X-ray diffraction and mass spectroscopy measurements, Mr K. Fukuoka, Dr Y. Murata, Dr W. Shinoda (T.I.Tech.) and Prof. S. Okazaki (T.I.Tech.) for small-angle X-ray 
scattering and molecular dynamics simulation, and Ms. J. Kawaguchi and Dr S. Lee for synthesis and CD measurements of CI2. The work was supported by the Grant-in-Aid for Scientific Research (B) (no. 08454227 and no. 0643060) from the Japanese Ministry of Education, Science, and Culture.

\section{REFERENCES}

1 J. C. Dore, J. Teixeira. Hydrogen-Bonded Liquids, NATO ASI Series C, Vol. 329. Kluwer Academic Publishers, Dordrecht (1991).

2 M.-C. Bellissent-Funel, J. C. Dore. Hydrogen Bond Networks, NATO ASI Series C, Vol. 435. Kluwer Academic Publishers, Dordrecht (1994).

3 J. C. Dore. In Water Science Reviews 1 (F. Franks, ed), Vol. 1, p. 3. Cambridge University Press, London (1985).

4 J. E. Enderby. In Aqueous Ionic Solutions (M.-C. Bellissent-Funel, G. E. Neilson, eds), NATO ASI Series C, Vol. 205, p. 129. Reidel, Dordrecht (1987).

5 M. Magini. X-Ray Diffraction of Ions in Aqueous Solutions: Hydration and Complex Formation. CRC, Florida (1988).

6 Y. Amemiya, J. Miyahara. Nature 336, 89 (1988).

7 K. Yamanaka, T. Yamaguchi, H. Wakita. J. Chem. Phys. 101, 9830 (1994).

8 A. K. Soper, R. N. Silver. Phys. Rev. Lett. 49, 471 (1982).

9 A. Rahman, F. H. Stillinger. J. Chem. Phys. 55, 3336 (1971).

10 R. L. McGreevy, L. Pusztai. Mol. Simul. 1, 359 (1988).

11 A. K. Soper. J. Chem. Phys. 101, 6888 (1994).

12 C. G. Gray, K. E. Gubbins. The Theory of Molecular Liquids. Oxford University Press, Oxford (1986).

13 A. K. Soper. Chem. Phys. 202, 295 (1996).

14 T. Sako, M. Sato. Industrial Materials (Kogyo Zairyo, Japanese) 44, 119 (1996).

15 T. Yamaguchi, C. J. Benmore, A. K. Soper. J. Chem. Phys. in press (2000).

16 T. Yamaguchi, K. Hidaka, A. K. Soper. Mol. Phys. 96, 1159 (1999); Erratum. Mol. Phys. 97, 603 (1999).

17 N. Nishi, S. Takahashi, M. Matsumoto, A. Tanaka, K. Muraya, T. Takamuku, T. Yamaguchi. J. Phys. Chem. 99, 462 (1995).

18 M. Matsumoto, N. Nishi, T. Furusawa, M. Saita, T. Takamuku, M. Yamagami, T. Yamaguchi. Bull. Chem. Soc. Jpn. 68, 1775 (1995).

19 T. Takamuku, M. Tabata, A. Yamaguchi, J. Nishimoto, M. Kumamoto, H. Wakita, T. Yamaguchi. J. Phys. Chem. 102, 8880 (1998).

20 T. Takamuku, A. Yamaguchi, M. Tabata, N. Nishi, K. Yoshida, H. Wakita, T. Yamaguchi, J. Mol. Liquids 83, 163 (1999).

21 K. Fukuoka. Master Thesis, Fukuoka University (1998); T. Yamaguchi, K. Fukuoka, Y. Murata, W. Shinoda, S. Okazaki. Manuscript in preparation.

22 N. Nishi, Y. Murata, T. Yamaguchi, S. Okazaki. Unpublished results.

23 P. Luo, R. L. Baldwin. Biochemistry 36, 8413 (1997).

24 S. Ludvigsen, H. Shen, M. Kjaer, J. C. Madsen, F. M. Poulsen. J. Mol. Biol. 222, 621 (1991).

25 L. S. Itzhaki, D. E. Otzen, A. R. Fersht, J. Mol. Biol. 254, 260 (1995).

26 J. Kawaguchi, T. Yamaguchi, S. Lee. Unpublished results. 